\title{
ERG Conductance Expression Modulates the Excitability of Ventral Horn GABAergic Interneurons That Control Rhythmic Oscillations in the Developing Mouse Spinal Cord
}

\author{
Francesco Furlan, ${ }^{1}$ Giuliano Taccola, ${ }^{2}$ Micaela Grandolfo, ${ }^{2}$ Leonardo Guasti, ${ }^{3}$ Annarosa Arcangeli, ${ }^{3}$ Andrea Nistri, ${ }^{2}$ and \\ Laura Ballerini ${ }^{1}$ \\ ${ }^{1}$ Physiology and Pathology Department, Center for Neuroscience B.R.A.I.N., University of Trieste, 34127 Trieste, Italy, ${ }^{2}$ Neurobiology Sector, International \\ School for Advanced Studies, 34014 Trieste, Italy, and SPINAL Project, Udine, ${ }^{3}$ Department of Experimental Pathology and Oncology, University of Firenze, \\ 50134 Firenze, Italy
}

\begin{abstract}
During antenatal development, the operation and maturation of mammalian spinal networks strongly depend on the activity of ventral horn GABAergic interneurons that mediate excitation first and inhibition later. Although the functional consequence of GABA actions may depend on maturational processes in target neurons, it is also likely that evolving changes in GABAergic transmission require fine-tuning in GABA release, probably via certain intrinsic mechanisms regulating GABAergic neuron excitability at different embryonic stages. Nevertheless, it has not been possible, to date, to identify certain ionic conductances upregulated or downregulated before birth in such cells. By using an experimental model with either mouse organotypic spinal cultures or isolated spinal cord preparations, the present study examined the role of the ERG current $\left(I_{\mathrm{K}(\mathrm{ERG})}\right)$, a potassium conductance expressed by developing, GABA-immunoreactive spinal neurons. In organotypic cultures, only ventral interneurons with fast adaptation and GABA immunoreactivity, and only after 1 week in culture, were transformed into high-frequency bursters by E4031, a selective inhibitor of $I_{\mathrm{K}(\mathrm{ERG})}$ that also prolonged and made more regular spontaneous bursts. In the isolated spinal cord in which GABA immunoreactivity and m-erg mRNA were colocalized in interneurons, ventral root rhythms evoked by NMDA plus 5-hydroxytryptamine were stabilized and synchronized by E4031. All of these effects were lost after 2 weeks in culture or before birth in coincidence with decreased $m$-erg expression. These data suggest that, during an early stage of spinal cord development, the excitability of GABAergic ventral interneurons important for circuit maturation depended, at least in part, on the function of $I_{\mathrm{K}(\mathrm{ERG})}$.
\end{abstract}

Key words: potassium channels; interneurons; spinal circuits; firing adaptation; ERG; inhibition; organotypic cultures; bursting

\section{Introduction}

Our understanding of spinal network development has advanced rapidly with the identification of the major factors responsible for motor circuit maturation (Jessel, 2000; Kiehn, 2006). Among them, an essential role is played by GABA-releasing interneurons that control wiring and network excitability within a restricted spatiotemporal window preceding birth (Caserta and Barker, 1994; Somogyi et al., 1995; Nishimaru et al., 1996; Gao et al., 2001; Nakayama et al., 2002; Allain et al., 2004, 2006). During embryogenesis, the role played by GABAergic neurons is finely tuned by activity-dependent programs that control GABA phenotype expression and repression in accordance with network operation (Feldblum et al., 1998; Branchereau et al., 2002). The action of GABA during development is often excitatory until shortly before birth, when locomotor networks acquire the ability

Received Sept. 15, 2006; revised Dec. 14, 2006; accepted Dec. 20, 2006.

This work was supported by FIRB projects to A.N. and L.B. (MUR and INFM), PRIN 2005, and a grant from the Friul Venezia Giulia Region. We are indebted to Sergio Graziosi for his computer and graphics assistance and to Beatrice Pastore for her assistance with tissue cultures.

Correspondence should be addressed to Dr. Laura Ballerini, Physiology and Pathology Department, Center for Neuroscience B.R.A.I.N., University of Trieste, via Fleming 22, 34127, Trieste, Italy. E-mail: ballerin@psico.units.it. D0I:10.1523/JNEUROSCI.4035-06.2007

Copyright $\odot 2007$ Society for Neuroscience $\quad$ 0270-6474/07/270919-10\$15.00/0 to generate alternating motor commands between flexor and extensor motoneurons. At this late stage of prenatal development, GABA-mediated excitation is replaced by synaptic inhibition mediated by glycine and/or GABA (Barbeau et al., 1999; Gao et al., 2001; Branchereau et al., 2002; Hanson and Landmesser, 2003), with surviving GABAergic neurons predominantly located in the dorsal horn (Phelps et al., 1999; Tran et al., 2003). Given the importance of GABA-dependent depolarizations as developmental signals (Ben-Ari, 2002; Owens and Kriegstein, 2002; Alvarez et al., 2005), it is necessary to clarify the processes regulating the embryonic GABAergic phenotype. Using organotypic slice cultures as an in vitro model system of spinal network development, we have recently reported that all embryonic GABAergic interneurons express $m$-erg genes and ERG proteins (Furlan et al., 2005). The erg genes code for a potassium current termed $I_{\mathrm{K}(\mathrm{ERG})}$ (Schwarz and Bauer, 2004) that possesses unusual kinetic characteristics, including fast inactivation with strong resurgent properties after hyperpolarization (Chiesa et al., 1997; Sacco et al., 2003). Because $I_{\mathrm{K}(\mathrm{ERG})}$ is amply expressed in developing neurons and progressively disappears during neuronal maturation (Arcangeli et al., 1997, 1998), it is a candidate mechanism for regulating the excitability of GABAergic interneurons before birth and thus for controlling the output of spinal networks. To 
investigate this possibility, we used organotypic cultures of the embryonic mouse spinal cord for patch-clamp recording from visually identified ventral interneurons. We first studied the cellular action of the selective $I_{\mathrm{K}(\mathrm{ERG})}$ inhibitor E4031 (Hirdes et al., 2005) on spike firing and spontaneous network discharges of interneurons immunocytochemically identified as GABAergic. Using the $I_{\mathrm{K}(\mathrm{ERG})}$ inhibitor E4031, we subsequently examined the contribution by $I_{\mathrm{K}(\mathrm{ERG})}$ to more complex patterns of rhythmic discharges involving phasic activation of GABAergic interneurons recorded from the mouse isolated spinal cord at different prenatal ages. Such an oscillatory behavior requires the coordinated activity of several spinal segments and emerges as motor network output (for review, see Kiehn, 2006). We observed the functional role of $I_{\mathrm{K}(\mathrm{ERG})}$ on network output as well as the coexpression of GABA and $m$-erg in certain interneurons. Our data thus indicate that $I_{\mathrm{K}(\mathrm{ERG})}$ is involved in the control of GABAergic interneuron excitability during spinal network antenatal development.

\section{Materials and Methods}

Preparation of organotypic cultures and acutely isolated spinal cords. Organotypic slice cultures of spinal cord and dorsal root ganglia (DRG) were obtained from mouse embryos (F1 hybrid C57BL6 $\times$ SJL) at days 12-13 of gestation, as described previously (Avossa et al., 2003; Furlan et al., 2005). Briefly, the fetuses were obtained by cesarean delivery from timedpregnant anesthetized mice (chloral hydrate $10.5 \%, 0.4 \mathrm{ml} / 100 \mathrm{~g}$, i.m.) that were subsequently killed by an intracardiac injection of chloral hydrate. This procedure is in accordance with the regulations of the Italian Animal Welfare Act, with the relevant European Union legislation and guidelines on the ethical use of animals, and is approved by the local Authority Veterinary Service. After isolation, fetuses were decapitated, and their backs were isolated and cut into $275-\mu \mathrm{m}$-thick transverse slices. The spinal cord with its DRG was then dissected from slices from low thoracic and high lumbar levels and fixed on a glass coverslip with reconstituted chicken plasma (Cocalico Biologicals, Reamstown, PA) clotted with thrombin (Sigma, Milan, Italy). The coverslips were inserted into plastic tubes with $1 \mathrm{ml}$ of medium. The medium contained 67\% DMEM (Invitrogen, Milan, Italy), $8 \%$ sterile water for tissue culture (Invitrogen), $25 \%$ fetal bovine serum (Invitrogen), and $25 \mathrm{ng} / \mathrm{ml}$ nerve growth factor (Alomone Laboratories, Jerusalem, Israel); osmolarity, 300 mOsm; pH 7.35. Each dissection yielded 30-50 slices that were kept in culture for 7-14 $\mathrm{d}$ before use. The tubes were kept in a roller drum rotating 20 times per hour in an incubator at $36.5^{\circ} \mathrm{C}$ in the presence of humidified atmosphere, with a concentration of $5.2 \% \mathrm{CO}_{2}$.

Several maturation processes take place in spinal slices grown for 1 or 2 weeks in vitro (Avossa et al., 2003; Rosato-Siri et al., 2004; Furlan et al., 2005). Our previous studies have indicated important differences in network activity between 1 week and 2 weeks of organotypic culturing (Ballerini and Galante, 1998; Rosato-Siri et al., 2004); therefore, these were taken as crucial time points to investigate the potential role of ERG in shaping circuit discharges.

To enable a meaningful comparison of the activity of cultured slice neurons with that of embryonic networks studied in isolated spinal preparations, we selected two developmental stages, namely embryonic day 15 (E15) and E19.5, that exhibited characteristic electrical discharges closely resembling those found at 7 or $14 \mathrm{~d}$ in vitro (DIV) (see Fig. 3). This observation is supported by previous studies of the developmental evolution of spinal network activity (Branchereau et al., 2002; Whelan 2003).

For experiments on isolated spinal cords, the tissues were removed from mouse embryos (E15-E19.5), which were delivered under urethane anesthesia $(0.2 \mathrm{ml}$, i.p., of a $10 \% \mathrm{w} / \mathrm{v}$ solution), and isolated from the mid-thoracic level to the cauda equina. At the end of the electrophysiological experiments, tissues were fixed with paraformaldehyde (4\% in Krebs' solution) and cryoprotected overnight in sucrose (30\% in Krebs' solution). They were then embedded in Tissue-Tek O.C.T. compound (Sakura Finetek Europe, Zoeterwoude, The Netherlands), frozen at $-20^{\circ} \mathrm{C}$, and finally sectioned $(25 \mu \mathrm{m})$ with a cryostat. The sections were mounted on gelatin-coated slides and dehydrated with ethanol, and after staining with Toluidine blue ( $1 \%$ in distilled water; see Fig. $5 A$ ), they were examined with a Zeiss Axioskop 2 MOT microscope ( $5 \times$ or $10 \times$; Carl Zeiss, Oberkochen, Germany).

Electrophysiological recordings and drug solutions. Recordings were performed from cultured spinal cord slices grown in vitro for 1 or 2 weeks (named 7 and 14 DIV). For each experiment, a coverslip with the culture was positioned in a Perspex chamber, mounted on an inverted microscope, and superfused with normal Krebs' solution containing (in $\mathrm{mM}$ ): $150 \mathrm{NaCl}, 4 \mathrm{KCl}, 1 \mathrm{MgCl}_{2}, 2 \mathrm{CaCl}_{2}, 10 \mathrm{HEPES}$, and 10 glucose. The $\mathrm{pH}$ was adjusted to 7.4 with $\mathrm{NaOH}$ (osmolarity, 305 mOsm).Current-clamp recordings in the whole-cell configuration (patch-clamp) were obtained at room temperature $(\mathrm{RT})\left(22 \pm 2^{\circ} \mathrm{C}\right)$ from visually identified ventral spinal neurons with pipettes $(4-6 \mathrm{M} \Omega$ ) filled with a solution of the following composition (in $\mathrm{mM}$ ): $120 \mathrm{~K}$-gluconate, $20 \mathrm{KCl}, 10 \mathrm{HEPES}, 10$ EGTA, $2 \mathrm{MgCl}_{2}$, and $2 \mathrm{Na}_{2} \mathrm{ATP}$; $\mathrm{pH} 7.35$ with $\mathrm{KOH}$. In accordance with previous investigations (Streit et al., 1991; Ballerini and Galante, 1998; Ballerini et al., 1999; Galante et al., 2000; Rosato-Siri et al., 2002; Avossa et al., 2003; Furlan et al., 2005), neurons were empirically termed interneurons when their cell body diameter was between 10 and $20 \mu \mathrm{m}$, thus making them clearly distinguishable from cells morphologically resembling motoneurons (30-60 $\mu \mathrm{m}$ diameter) and immunoreactive to the motoneuron marker SMI32 (supplemental Fig. 2, available at www.jneurosci.org as supplemental material).Unlike interneurons, motoneurons generated long processes that often terminated on the muscle fibers distributed laterally to the slice (Ballerini et al., 1999; Avossa et al., 2003). In a set of experiments $(n=8)$, interneuron morphology was confirmed by direct identification after filling with neurobiotin (2\%; Vector Laboratories, Burlingame, CA) (see Fig. $2 C$ and below). Interneurons could also be distinguished from DRG neurons (Avossa et al., 2003) that possessed a large cell body (40 $\mu \mathrm{m}$ diameter) with just one or two large processes emanating from it (Streit et al., 1991). A detailed description of the identification of ERG-positive neurons as interneurons has been reported (Furlan et al., 2005), and it is confirmed by the high likelihood of colocalization of ERG and GABA, a typical transmitter of interneurons (Furlan et al., 2005) (see Fig. 7). The term "interneuron" does not exclude, however, the possibility of including projection or propriospinal neurons within the tested cell population, although the restricted size of the network and its very thin layer structure would have predominantly comprised local neurons. Electrophysiological responses were amplified (Axopatch 1-D; Axon Instruments, Foster City, CA), displayed on a chart recorder, digitized, and recorded at $10 \mathrm{kHz}$ with the pCLAMP software (version 8.1; Axon Instruments) for later analysis. Bridge balancing was monitored continuously. Action potential (AP) discharge patterns were investigated by delivering depolarizing current steps of variable intensity while keeping the cells at $-60 \mathrm{mV}$ resting potential with steady intracellular current injection (usually $50 \mathrm{pA}$ ). The amount of adaptation $\left(A_{(t)}\right)$ was quantified according to $A_{(t)}=100\left(f_{\max }-f_{(i)}\right) / f_{\max }$, where $f_{\max }$ is the firing frequency during the first $100 \mathrm{~ms}$ at the beginning of each pulse, and $f_{(i)}$ is the firing frequency measured every $100 \mathrm{~ms}$ along the pulse. For each cell, the area and duration of single spikes were measured from $>100$ spikes (Clampfit software, version 9,2; Axon Instruments) evoked by depolarizing current pulses of identical amplitude at the same membrane potential level and averaged. For "adapting" neurons, only the first two APs were sampled for this analysis. The total firing frequency of APs for each neuron was calculated on a sample of $>5 \mathrm{~min}$ continuous recording. In each cell, the intraburst firing frequency was calculated by measuring spikes within at least 15 individual bursts and then averaging data.

On a sample of 18 organotypic mouse spinal cord slices from $10 \mathrm{cul}-$ ture series, interneuron resting membrane potential did not differ between 7 $(-55 \pm 3 \mathrm{mV})$ and $14(-58 \pm 2 \mathrm{mV})$ DIV. Input resistance, measured by injecting hyperpolarizing current steps $(-100 \mathrm{pA} ; 100 \mathrm{~ms})$, significantly $(p<0.001)$ decreased from $312 \pm 25 \mathrm{M} \Omega(7 \mathrm{DIV})$ to $256 \pm 35 \mathrm{M} \Omega(14$ DIV), probably because of neuronal growth (Avossa et al., 2003).

In the present report, voltage-clamp recordings were performed on a random sample of 11 neurons at 7 DIV to confirm the presence of $I_{\mathrm{K} \text { (ERG) }}$ as detailed in our previous study (Furlan et al., 2005). Because ERG channel conductance is directly related to $\left[\mathrm{K}^{+}\right]_{\mathrm{o}}$, we used the standard protocol to study $I_{\mathrm{K}(\mathrm{ERG})}$ with a modified Krebs' solution containing 
high $\left[\mathrm{K}^{+}\right]_{\mathrm{o}}$ (Furlan et al., 2005; Hirdes et al., 2005). Osmolarity was kept constant by lowering the $\left[\mathrm{Na}^{+}\right]_{\mathrm{o}}$. In this solution, the divalent cation concentration was also changed ( $\left.1 \mathrm{~mm}\left[\mathrm{Ca}^{2+} / 2 \mathrm{~mm} \mathrm{Mg}^{2+}\right]\right)$ to minimize spontaneous miniature postsynaptic currents. The external Krebs' solution contained (in mM): $114 \mathrm{NaCl}, 40 \mathrm{KCl}, 1 \mathrm{CaCl}_{2}, 2 \mathrm{MgCl}_{2}, 10 \mathrm{HEPES}$, and 10 glucose. To this solution was added tetrodotoxin ( $1 \mu \mathrm{M}$; Affiniti) to block $\mathrm{Na}^{+}$channels. In the present study, 7 DIV ventral interneurons ( 7 of 11 cells) produced an inward current when repolarized to $-140 \mathrm{mV}$ for $250 \mathrm{~ms}$ after a conditioning step to $0 \mathrm{mV}$ for $15 \mathrm{~s}$. Such a current was fully blocked by E4031 (Alomone Laboratories) [used at a saturating concentration of $1 \mu \mathrm{M}$ to ensure full, rapid block of $I_{\mathrm{K}(\mathrm{ERG})}$ (Schwarz and Bauer, 1999; Bauer and Schwarz, 2001; Wimmers et al., 2001; Hirdes et al., 2005)] and was absent when preceded by a $15 \mathrm{~s}$ step to $-70 \mathrm{mV}$. These properties are typical of $I_{\mathrm{K} \text { (ERG) }}$ (Sacco et al., 2003; Furlan et al., 2005; Hirdes et al., 2005), which is usually expressed by $\sim 70 \%$ of interneurons at this developmental stage (Furlan et al., 2005).

Acutely isolated spinal cords were superfused continuously $(6.5 \mathrm{ml} /$ min) with oxygenated $\left(95 \% \mathrm{O}_{2} / 5 \% \mathrm{CO}_{2}\right)$ Krebs' solution of the following composition (in $\mathrm{mm}$ ): $113 \mathrm{NaCl}, 4.5 \mathrm{KCl}, 1 \mathrm{MgCl}_{2}, 2 \mathrm{CaCl}_{2}, 1$ $\mathrm{NaH}_{2} \mathrm{PO}_{4}, 25 \mathrm{NaHCO}_{3}$, and 11 glucose; $\mathrm{pH}$ 7.4. Electrophysiological recordings were performed from ventral roots (VRs) (Kiehn, 2006; Nistri et al., 2006) by means of suction electrodes; fictive locomotor patterns were obtained by recording the activity from left and right L2 VRs (mostly flexor motor-pool commands to the hindlimbs) and left and right L5 VRs (mostly extensor motor-pool commands to the hindlimbs) (Kiehn and Kjaerulff, 1998; Nistri et al., 2006). A typical indicator of fictive locomotion (Butt et al., 2002) is a pattern of discharge alternation between homosegmental left and right VRs and between L2/L5 VRs on the same side. All experiments were performed at RT. E4031 (Alomone Laboratories), NMDA, 5-HT, and CNQX (Tocris, Bristol, UK), and bicuculline methiodide (Sigma) were bath applied.

Non-radioactive in situ hybridization and immunofluorescence. Mouse spinal cords were removed from both $\mathrm{E} 15(n=3)$ and E19.5 $(n=3)$ embryos, without dissecting the column to maximally preserve structure. Specimens were fixed in $4 \%$ paraformaldehyde for $15 \mathrm{~h}$ at $4^{\circ} \mathrm{C}$, washed, and dehydrated with alcohol. Sections were dewaxed with xylene, rehydrated, washed in PBS, and blocked with 5\% normal goat serum (Sigma) in $0.2 \%$ PBS-Triton X-100 ("blocking solution 1"). Sections were subsequently incubated overnight with or without monoclonal GABA antibody (Chemicon, Milan, Italy) and diluted (1:600) in blocking solution 1. After three washes with PBS, sections were incubated for $2 \mathrm{~h}$ with goat antimouse fluorescein isothiocyanate (FITC)-conjugated secondary antibody (Chemicon) diluted (1:400) in blocking solution 1. After washing in PBS, sections were coverslipped with glycerol ( $\mathrm{pH}$ 8.6)/ PBS (9:1) containing $2.5 \%(\mathrm{w} / \mathrm{v})$ 1,4-diazabicyclo-[2,2,2] octane (DABCO) (Sigma).

Double non-radioactive in situ hybridization (NR-ISH) (Furlan et al., 2005) and immunofluorescence were performed to test the coexpression of $m$-erg genes and GABA. For this purpose, the monoclonal anti-GABA antibody was added together with the alkaline phosphatase-conjugated sheep anti-digoxygenin antibody (Roche, Milan, Italy), followed by incubation with goat anti-mouse secondary FITC-conjugated antibody. Digoxygenin detection was then performed with the alkaline phosphatase substrate nitroblue tetrazolium/5-bromo-4-chloro-3-indolyl phosphate (Roche). Slides were mounted as above. Labeling was performed on at least five sections per slide, and each slide was tested in duplicate. Because we observed the same cells in the same sections, this approach avoided the risk of erroneous colocalization of markers had different sections been used. Images were acquired with a Leica DMR microscope equipped with a Leica DC200 digital camera (Leica Microsystems, Nussloch, Germany).

For immunofluorescence experiments (Furlan et al., 2005), cultures were incubated in $1 \%$ fetal calf serum, $5 \%$ bovine serum albumin, and $0.3 \%$ Triton X-100 in PBS (blocking solution) for $30 \mathrm{~min}$ at RT and subsequently incubated with the rabbit polyclonal anti-GABA antibody (Sigma; 1:250) for $16 \mathrm{~h}$ at $4^{\circ} \mathrm{C}$ in a humid chamber. Cultures were incubated with Alexa 594-labeled secondary antibody (Molecular Probes, Eugene, OR; 1:500), and streptavidin FITC (Pierce, Rockford, IL; 1:100) in blocking solution for $2 \mathrm{~h}$ at RT. After washing in PBS, cultures were mounted in glycerol ( $\mathrm{pH} 8.6$ )/PBS (9:1) containing 2.5\% (w/v) DABCO
(Sigma) to prevent fluorescence fading. The samples were viewed with a Zeiss Axioskop microscope equipped with a Cool Snap RS Photometrics camera (Roper Scientific, Tucson, AZ), and the pictures were obtained with Metamorphe software (Molecular Devices Corporation, Palo Alto, CA).

Additional double immunofluorescence experiments were performed to check the potential expression of ERG proteins by glial fibrillary acidic protein (GFAP)-positive cells (supplemental Fig. 1, available at www.jneurosci.org as supplemental material) or the possibility of nonspecific staining of motoneurons by the GABA antibody (supplemental Fig. 2, available at www.jneurosci.org as supplemental material). For this first goal, fixed cultures were incubated in 3\% fetal calf serum, 3\% bovine serum albumin, and $0.3 \%$ Triton X-100 in PBS (blocking solution) for 30 min at RT and subsequently incubated with the following antibodies: mouse monoclonal anti-GFAP (1:200 dilution; Sigma) or the in-housegenerated rabbit polyclonal anti-HERG1 (Avossa et al., 2003). For the second goal, fixed cultures were incubated with the following: mouse monoclonal SMI32 (1:200 dilution; Sternberger Monoclonal, Lutherville, $\mathrm{MD}$ ) directed toward the nonphosphorylated epitope of neurofilament $\mathrm{H}$ to identify motoneurons (Avossa et al., 2003; 2006) and rabbit polyclonal anti-GABA antibody (1:250; Sigma). The incubation was for $16 \mathrm{~h}$ at $4^{\circ} \mathrm{C}$ in a humid chamber. After washing, cultures were incubated with the corresponding Alexa 594-labeled or Alexa 488-labeled (Molecular Probes) secondary antibodies (1:300) for $2 \mathrm{~h}$ at RT. After washing in PBS, cultures were mounted in glycerol ( $\mathrm{pH} 8.6) / \mathrm{PBS}$ (9:1) containing $2.5 \%(\mathrm{w} / \mathrm{v})$ DABCO (Sigma) to prevent fluorescence fading. Images were obtained with a TCS SP2 Leica confocal microscope. Five organotypic slices (7-14 DIV) were used, of which 15 randomly chosen ventral horn fields were examined in detail.

Statistical analysis. Ventral interneuron recordings were obtained from 65 culture series. Results are presented as mean \pm SD, with $n=$ number of neurons, unless stated otherwise. Statistical analysis was performed with the Student's $t$ test (parametric data) or the ANOVA test (nonparametric data). Differences were considered significant at $p<0.05$.

The analysis of rhythms evoked by isolated spinal cords was performed as indicated by Taccola and Nistri (2004). The discharge period ( T) was measured as the time between the onset of two subsequent oscillations (calculated as an average of 20 cycles). All data were expressed as mean \pm $\mathrm{SD}$, with $n=$ number of experiments. The Rayleigh test and circular statistics were used to analyze the strength of phase coupling between roots (Drew and Doucet, 1991), as reported previously (Taccola and Nistri, 2004). The length of vectors in the polar plots quantifies the strength of signal coupling, whereas the direction shows the concentration of phase values around the mean phase. The $\Phi$ value, expressed in angular degrees, corresponds to the time span from the top of a cycle in one root to the top of the corresponding cycle in the other root, divided by the period. A $\Phi=180^{\circ}$ means that the two cycles are completely alternating, whereas a $\Phi$ value of $0^{\circ}$ or $360^{\circ}$ describes a fully coincident phase (Kjaerulff and Kiehn, 1996). For comparisons between two groups, the following tests were performed depending on data: Student's $t$ test (paired or unpaired) for normally distributed data and Mann-Whitney test for non-normally distributed data. For multiple comparisons, ANOVA tests were used (ANOVA on ranks, in the case of nonparametric data), followed by a post hoc test (Tukey's test).

\section{Results}

\section{Spinal ventral interneurons expressed different discharge patterns during maturation in culture}

During antenatal development, the spinal cord transiently expresses, within a definite time window, three ERG proteins (ERG1a, ERG2, and ERG3) that are localized to GABAergic interneurons (Avossa et al., 2003; Furlan et al., 2005) and contribute to the functional manifestation of $I_{\mathrm{K}(\mathrm{ERG})}$ (Furlan et al., 2005). Because the role of $I_{\mathrm{K}(\mathrm{ERG})}$ in the control of membrane excitability of ventral interneurons is unknown, we first investigated the age-dependent distribution of interneuron excitability in organotypic slice cultures to understand how $I_{\mathrm{K}(\mathrm{ERG})}$ might modify it. To this end, we studied the evoked firing properties of ventral interneurons at 7 and 14 DIV. Firing patterns were induced by 
depolarizing current steps (500 ms duration; 0.1-0.2 nA amplitude) (Fig. 1) in 54 interneurons recorded at 7 and 14 DIV ( $n=26$ and 28, respectively) in the presence of CNQX (10 $\mu \mathrm{M} ; 10 \mathrm{~min})$ and bicuculline $(20 \mu \mathrm{M} ; 10 \mathrm{~min})$ to prevent the occurrence of spontaneous synaptic events. All neurons were held at $-60 \mathrm{mV}$ membrane potential, a value close to their resting potential (see Materials and Methods). Cells were kept at $-60 \mathrm{mV}$ (because their spike threshold was approximately -50 $\mathrm{mV}$ ) to prevent spontaneous firing (Szucs et al., 2003), which obscures stimulusevoked responses.

We could identify five different classes of interneurons on the basis of their firing pattern (Fig. $1 A, B$ ) (Prescott and de Koninck, 2002; Szucs et al., 2003; Theiss and Heckman, 2005; Lu et al., 2006): (1) "tonic" cells, which continuously fired APs without apparent accommodation; (2) "adapting" cells, which discharged an early burst of APs followed by adaptation; (3) "delay" cells, which generated APs after a lag; (4) "irregular" cells without a discernible pattern of AP discharge; and (5) "transient" cells, which generated a single AP only. Interestingly, the distribution of the five neuronal classes was maturation dependent.

As shown in Figure 1, $A$ and $C$, at 7 DIV, cells belonged to tonic, adapting, delay, and irregular classes. At this age, most $(60 \%)$ of the recorded ventral interneurons were of the adapting type, whereas the others were equally distributed in the remaining 40\%. As shown in Figure 1, $C$ and $D$, the distribution-profile of interneuron types was clearly changed after spinal slice maturation. In fact, at 14 DIV, most (60\%) of the recorded cells were tonic, and only $15 \%$ were represented by adapting neurons. Adapting cells at 14 DIV displayed $A_{\text {(t) }}$ values comparable with those detected at 7 DIV (on average, $71 \pm 15$ and $65 \pm 5 \%$ at 7 and 14 DIV, respectively), whereas they differed in terms of spikes/pulse (on average, $4 \pm 1$ and $10 \pm 2$ spikes per step at 7 and $14 \mathrm{DIV}$, respectively; $n=4)($ Fig. $1 A, B)$. Delay interneurons were $15 \%$ of the population, whereas transient cells were $10 \%$ (irregular cells could not be detected). It is important to note that, in each class, firing frequency at 14 DIV was higher than at 7 DIV (Fig. $1 A, B$ ). In particular, AP frequency in tonic cells increased from $29 \pm 0.7 \mathrm{~Hz}$ to $41 \pm 4 \mathrm{~Hz}$ (7 and 14 DIV, respectively; $n=330$ spikes; $p<0.05)$. In the same cell group, AP area decreased from $280 \pm 7$ to $209 \pm 4 \mathrm{mV} / \mathrm{ms}$ ( 7 and 14 DIV, respectively; $n=330 ; p<0.001$ ) together with AP duration (from $4.2 \pm 0.06$ to $3.5 \pm 0.05 \mathrm{~ms} ; 7$ and $14 \mathrm{DIV}$, respectively; $n=330 ; p<0.001)$.

It is noteworthy that a similar classification of firing patterns of ventral interneurons (with a strong preponderance of tonic cells) (Szucs et al., 2003; Theiss and Heckman, 2005) has been reported for newborn spinal cord preparations, indicating that the observed differences were not caused by culturing conditions.
$I_{\text {K(ERG) }}$ selectively affected firing of adapting neurons at 7 DIV We next tested E4031, a selective blocker of $I_{\mathrm{K}(\mathrm{ERG})}$ (Furlan et al., 2005; Hirdes et al., 2005) on all neuron classes at 7 and 14 DIV ( $n=26$ and 28, respectively) in the presence of CNQX and bicuculline to inhibit synaptic transmission. E4031 evoked no change in resting membrane potential of these neurons. Although tonic, delay, irregular, or transient neurons were never affected by E4031, at 7 DIV pharmacological removal of $I_{\mathrm{K}(\mathrm{ERG})}$ converted adapting neurons to tonic ones (Fig. $2 A$ ), as quantified by the strong decrease in $A_{(\mathrm{t})}$ value (from $71 \pm 15$ to $18 \pm 7 \% ; n=12$ ) (Fig. $2 B$ ). On the contrary, adapting ventral neurons at $14 \mathrm{DIV}$ were never affected by E4031 application $\left(A_{(\mathrm{t})}\right.$ in the presence of E4031 remained, on average, $63 \pm 4 \%$ ). Regardless of the action by E4031 on spike adaptation, its application did not affect AP area and duration $(271 \pm 15 \mathrm{mV} / \mathrm{ms}$ and $4.3 \pm 0.04 \mathrm{~ms}$ and $218 \pm$ $13 \mathrm{mV} / \mathrm{ms}$ and $3.4 \pm 0.02 \mathrm{~ms}$ area and duration in E4031 at 7 and 14 DIV, respectively; $n=120$ spikes).

To further identify such E4031-sensitive neurons (at 7 DIV), at the end of the electrophysiological recording protocol, a sample $(n=8)$ of these adapting interneurons was filled with neuro- 


\section{A}
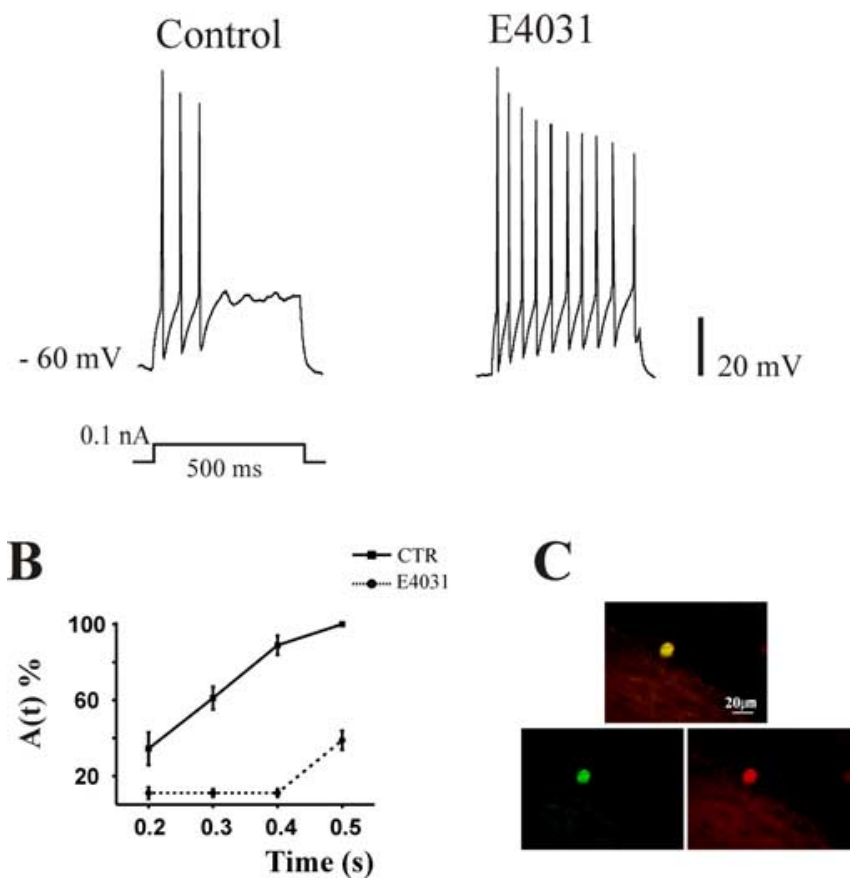

Figure 2. Effects of E4031 on adapting cells at 7 DIV. A, Current-clamp recordings of an adapting interneuron in control solution (left trace) and after $10 \mathrm{~min}$ application of $1 \mu \mathrm{m}$ E4031 (right trace). Note the removal of adaptation by pharmacological block of $I_{\text {K(ERG) }} \cdot \boldsymbol{B}$, The plot summarizes percentage changes in adaptation quantified as $A_{(T)}$ in control (CTR, filled square) and in the presence of E4031 (filled circles). Data are pooled from 12 cells. Note the presence of strong adaptation in CTR neurons that was significantly removed by the application of E4031. C, Example of positive double-staining of a recorded adapting cell for neurobiotin (green) and GABA (red) and their merged image (top). The same cell as in $A$ is shown.

biotin (Fig. 2C, green) and stained for GABA immunofluorescence as exemplified in Figure $2 C$ (red; merged images are shown in yellow). Previous studies have shown that such morphologically and functionally identified neurons express GAD67 (67$\mathrm{kDa}$ isoform of glutamic acid decarboxylase) (Avossa et al., 2003) in keeping with their GABAergic phenotype. All cells showed costaining indicating that $I_{\mathrm{K}(\mathrm{ERG})}$-dependent adaptation was typically found in GABAergic interneurons. This result accords with former evidence indicating that, in spinal cultured slices, ERGla, ERG2, and ERG3 are expressed by all GABA-positive neurons and that this expression is functionally manifested by E4031sensitive $I_{\mathrm{K}(\mathrm{ERG})}$ (Furlan et al., 2005). Indeed, 14 DIV ventral neurons that do not express ERG proteins cannot show $I_{\mathrm{K}(\mathrm{ERG})}$ and are unresponsive to E4031 (Furlan et al., 2005).

\section{Effects of E4031 on spontaneous firing activity in cultured spinal ventral interneurons}

We next explored the contribution by $I_{\mathrm{K}(\mathrm{ERG})}$ to network activity in standard Krebs' solution without pharmacological block of synaptic transmission. At $-60 \mathrm{mV}$ membrane potential (obtained with steady current injection; $-50 \pm 15 \mathrm{pA}$ ), interneurons ( $90 \%$ of slices) at 7 DIV displayed spontaneous bursts ( $0.03 \pm$ $0.005 \mathrm{~Hz}$ frequency; $3.2 \pm 1$ s duration) (Fig. $3 A$, top) comprising summated synaptic potentials with superimposed highfrequency firing. Each burst was followed by $19 \pm 2 \mathrm{~s}$ intervals that were usually free from spikes (Fig. $3 A$, top). The total firing frequency was $0.36 \pm 0.07 \mathrm{~Hz}(n=18)$, whereas the intraburst firing frequency was, on average, $3.54 \pm 0.07 \mathrm{~Hz}(n=10)$. After 2 weeks in culture, $75 \%$ of slice preparations lacked burst-driven
A

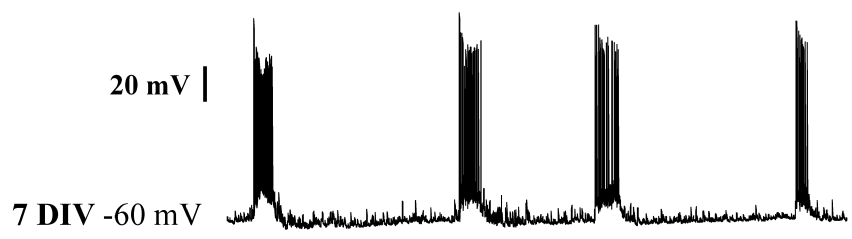

E 15 VRrL5

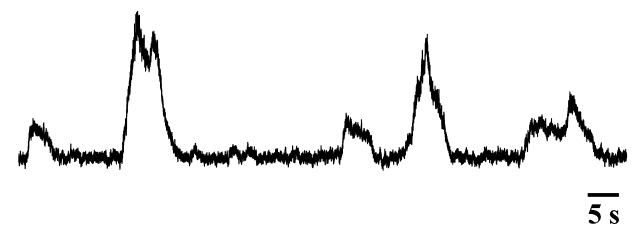

B

14 DIV

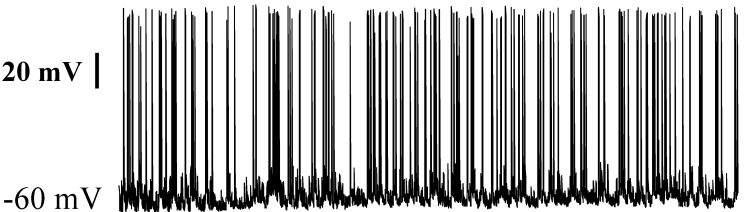

E 19.5 VRrL5

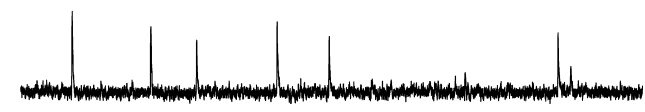

Figure 3. Network-driven activity recorded from ventral interneurons after 1 and 2 weeks in slice cultures or recorded from E15 and E19.5 spinal cords. $\boldsymbol{A}, \boldsymbol{B}$, Spontaneous activity in control solution is shown both at 7 DIV $(\boldsymbol{A}$, top) and E15 ( $\boldsymbol{A}$, bottom) and at $14 \mathrm{DIV}$ ( $\boldsymbol{B}$, top) and E19.5 ( $\boldsymbol{B}$, bottom). Note the developmental changes in firing patterns from bursting to nonbursting. In neurons recorded at $14 \mathrm{DIV}$, this switch in network activity was accompanied by a large increase in firing frequency. VRrL5, Right L5 VR.

firing because APs were tonically generated on a background of spontaneous synaptic activity (Fig. $3 B$, top). The overall firing frequency at 14 DIV was $0.88 \pm 0.12 \mathrm{~Hz}$, a value significantly higher than that recorded at 7 DIV $(p<0.001 ; n=18)$.

Because ERG proteins are primarily localized to embryonic GABAergic interneurons (Avossa et al., 2003; Furlan et al., 2005), we were interested in understanding whether GABAergic interneurons might have regulated network excitability via $I_{\mathrm{K}(\mathrm{ERG})}$. Thus, whole-cell current-clamp recordings were performed from ventral spinal neurons grown in organotypic cultures at 7 and 14 DIV before and after adding E4031 ( $1 \mu \mathrm{M} ; 10 \mathrm{~min})$. On spontaneously bursting neurons, E4031 increased total firing frequency to $206 \pm 26 \%$ (Fig. $4 A)(n=8 ; p<0.05)$. This boost in excitability comprised a significant increase in intraburst firing frequency $(4.23 \pm 0.8 \mathrm{~Hz} ; n=8 ; p<0.05)$ together with the appearance of interburst APs (Fig. $4 A$, bottom). In addition, E4031 caused a significant increase in burst duration $(4.25 \pm 1.27 \mathrm{~s} ; n=$ $10 ; p<0.05)$ and a small, although significant, reduction in burst frequency $(0.02 \pm 0.003 \mathrm{~Hz} ; n=10 ; p<0.05)$. With the exception of two interneurons that were depolarized $(4 \mathrm{mV})$ by E4031 (potential corrected back to $-60 \mathrm{mV}$ by injection of hyperpolar- 
izing current), the other cells $(n=8)$ did not show any change in resting potential or resistance. On neurons tested at $14 \mathrm{DIV}$ $(n=8)$, E4031 had no effect on resting membrane potential, input resistance, or network-driven firing activity (Fig. $4 B$ ).

Because in the mammalian nervous system ERG can also be expressed by glia (Papa et al., 2003), we investigated whether ERG could be found in glial cells (identified on the basis of their GFAP immunostaining) of organotypic slices. Supplemental Figure 1 (available at www.jneurosci.org as supplemental material) shows full segregation between ERG1- and GFAP-expressing cells (a result confirmed in five slices at 7 or 14 DIV), indicating that the functional effects caused by the ERG inhibitor could not be mediated indirectly by ERG expressing glia.

\section{Effects of E4031 on the isolated embryonic spinal cord}

Because the strongest expression of $m$-erg genes in the ventral horn is at approximately E15, with subsequent, large downregulation near birth (Furlan et al., 2005), we investigated whether E4031 could affect the integrated network patterns that can be observed in isolated spinal cord preparations at E15 or E19.5 (Fig. $5 A, B)$. For this purpose, we measured spontaneous VR discharges as well as rhythmic oscillations induced by coapplication of NMDA and 5-HT (Vinay et al., 2002). Note that, at E15, spinal cords produced typical VR bursting (Fig. $3 A$, bottom) similar to the rhythmic discharges observed in cultures at 7 DIV. At E19.5, the VR discharges became sporadic and lacked bursting patterns (Fig. 3B, bottom). At 14 DIV, ventral interneurons had also lost their rhythmic activity, which was replaced by high-frequency irregular firing (Fig. 3B). These observations suggest that, from the point of view of spontaneous electrical activity, the E15 pattern resembled the 7 DIV pattern, and that the late irregular activity of interneurons was not associated with any rhythmic discharge of motoneurons in the isolated spinal cord.

Figure $5 C$ shows how the period of spontaneous VR activity (E15) was decreased (by 39\%) by E4031, together with $80 \mu \mathrm{V} \mathrm{VR}$ depolarization. Pooled data from four E15 preparations indicated that E4031 diminished the spontaneous discharge period (by $40 \pm 20 \%$; $p<0.03$ ) together with a modest VR depolarization $(47 \pm 25 \mu \mathrm{V})$. On the other hand, the same drug did not affect spontaneous VR discharges from E19.5 preparations [period values $(T)$ were $26 \pm 15 \mathrm{~s}$ in control solution and $30 \pm 15 \mathrm{~s}$ in the presence of $\mathrm{E} 4031 ; p>0.5 ; n=3$ ].

As shown in Figure 5, D and D1, for an E15 spinal cord, NMDA $(5 \mu \mathrm{M})$ and 5-HT $(10 \mu \mathrm{M})$ evoked a pattern of rhythmic oscillations $(T=17 \pm 2 \mathrm{~s}$; duration $=12 \pm 3 \mathrm{~s}$ ) replacing ongoing spontaneous activity. Subsequent addition of $1 \mu \mathrm{M}$ E4031 hyperpolarized VRs $(110 \mu \mathrm{V})$ with a concomitant increase in period $(T=179 \pm 29 \%)($ Fig. $5 D, D 2)$ and duration $(128 \pm 40 \%)$ (Fig. 5D,D2). In NMDA plus 5-HT solution, oscillations lacked alternation between right and left L5 VRs $\left(\Phi=142 \pm 40^{\circ}\right)$ (Fig. $5 D, D 1)$ and were poorly phase-coupled, as exemplified by the small value of the vector in the polar plot of Figure $5 E$ and confirmed by its low value with the Rayleigh test $(r=0.78)$. Subsequent application of E4031 (Fig. 5D,D2) elicited a synchronous pattern $\left(\Phi=35 \pm 38^{\circ} ; r=0.82\right.$ ) (Fig. $\left.5 F\right)$.

In a sample of seven E15 preparations, the irregular nature of
B

14 DIV

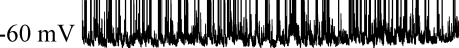

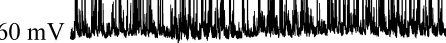
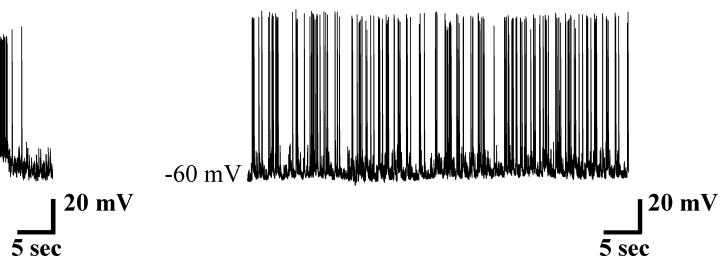

\rfloor$^{20 ~ m V}$

Figure 4. E4031 effects on network excitability of spinal slices at 7 and 14 DIV. A, Top, Bursting activity in control solution recorded from a 7 DIV interneuron located in the ventral area of a spinal slice in culture. Bottom, The application (10 min) of E4031 $(1 \mu \mathrm{m})$ prolonged burst duration with a reduction in burst frequency. $\boldsymbol{B}$, Representative tracings of spontaneous activity, recorded at 14 DIV in control condition (top trace) and in the presence of $1 \mu \mathrm{m}$ E4031 (10 min; bottom trace). Note that at 14 DIV, E4031 failed to evoke any increase in firing activity and that E4031 significantly increased firing frequency at 7 DIV only.

NMDA plus 5-HT-evoked oscillations was demonstrated by the large spreading of phase values around the mean $\Phi(101 \pm 71)$, indicating that neither alternation nor synchronicity appeared between homolateral and homosegmental pairs of lumbar VRs. E4031 did not affect the value of the Rayleigh vector $(r=0.67 \pm$ $0.35)$, but it significantly decreased $\Phi$ to $-10.9 \pm 92.5(p<$ $0.05)$. Pooled data confirmed that E4031 produced a statistically significant increase in period $(T=156 \pm 62 \%$; $p<0.03)$ and duration ( $142 \pm 62 \%$; $p<0.05$; control values were $11.3 \pm 3.8$ and $6.9 \pm 2.9$ s, respectively; $n=6$ ). This effect by E4031 was unrelated to VR polarity changes, because two preparations were depolarized $(82 \pm 3 \mu \mathrm{V})$ and four were hyperpolarized $(-185 \pm$ $70 \mu \mathrm{V})$. Thus, pharmacological block of $I_{\mathrm{K}(\mathrm{ERG})}$ at E15 was followed by stabilization of drug-evoked rhythmicity at a lower rate of synchronized activity.

This effect of E4031 disappeared when alternating fictive locomotor patterns emerged at E19.5 (Whelan, 2003). This phenomenon is exemplified in Figure 6 (left), which shows that NMDA plus 5-HT induced fictive locomotor activity consisting of pattern alternation between homosegmental left and right VRs and between related flexor (L2) and extensor (L5) motor pools on the same side. Polar plots (left, bottom) show the double alternation and the phase coupling between left L2 and L5 ( $\Phi=161 \pm$ $16^{\circ} ; n=20$ cycles; $\left.r=0.96\right)$ and between right and left L5 ( $\Phi=$ $201 \pm 25^{\circ} ; n=20$ cycles; $r=0.91$ ). Figure 6 (right) shows that E4031 did not affect the rhythm, which maintained a similar period ( $T=2.5 \pm 0.2 \mathrm{vs} 2.7 \pm 0.2 \mathrm{~s}$ in NMDA plus 5 -HT alone) and a similar duration $(1.5 \pm 0.1 \mathrm{~s}$ with respect to $1.6 \pm 0.2 \mathrm{~s}$ in NMDA plus 5-HT alone). Furthermore, E4031 did not change the characteristic double alternation of the fictive locomotor rhythm as reported by the polar plots in Figure 6 (right, bottom), showing a $\Phi$ value of $166 \pm 20^{\circ}(n=20$ cycles; $r=0.94)$ for homolateral and a $\Phi$ value of $199 \pm 19^{\circ}(n=20$ cycles; $r=0.95)$ for homosegmental pairs of VRs. Pooled data from three preparations indicated that, at E19.5, E4031 changed neither period $(T=99 \pm$ $11 \% ; n=3)$ nor duration ( $99 \pm 14 ; n=3$ ) of fictive locomotion.

\section{m-erg genes in the embryonic spinal cord}

The developmental stage at which blocking $I_{\mathrm{K}(\mathrm{ERG})}$ yielded significant changes in spinal network activity corresponded to the expression of $m$-erg genes in the ventral spinal cord in vivo (Furlan 


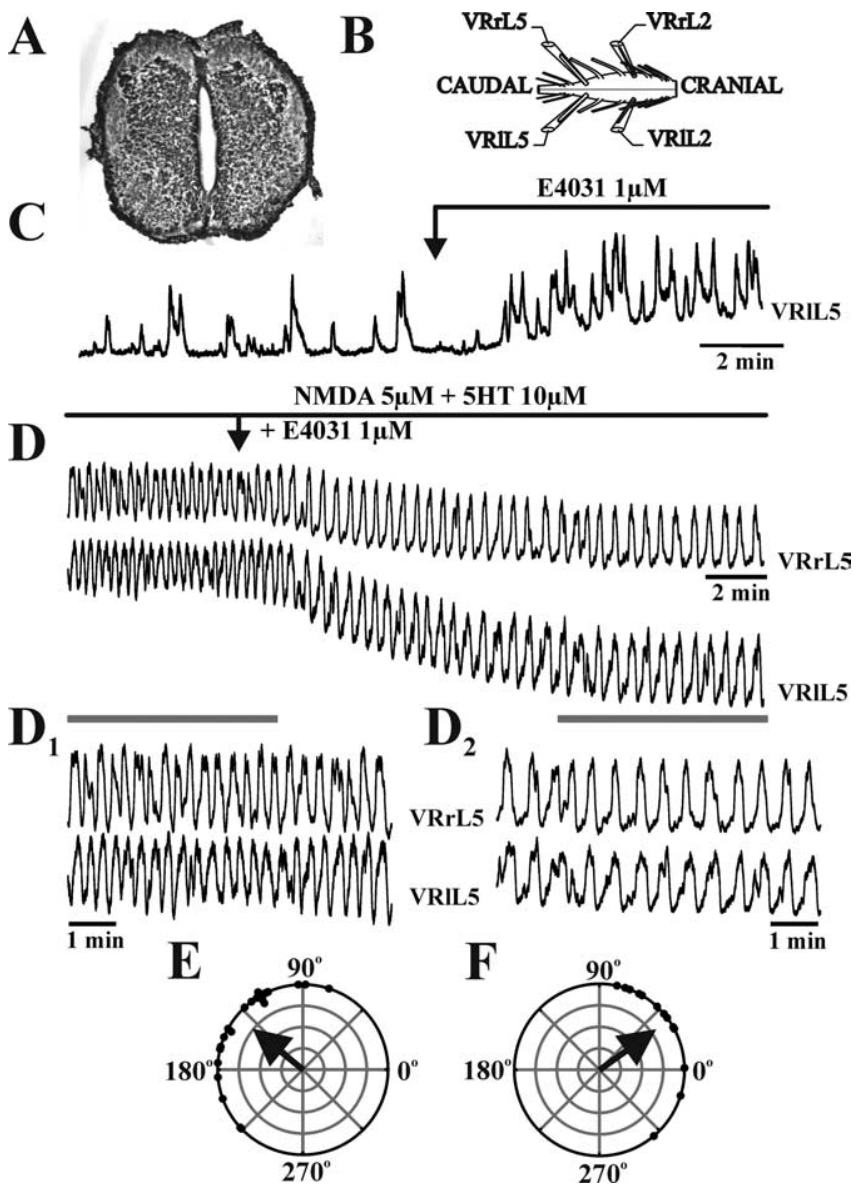

Figure 5. Effects of E4031 on the spinal cord isolated from a 15-d-old mouse embryo. Histological section of E15 mouse lumbar spinal cord $(\boldsymbol{A})$ and schematic diagram of the experimental setup for VR recordings (B).C, Spontaneous activity recorded from E15 VR in control and after $10 \mathrm{~min}$ application of $\mathrm{E} 4031(1 \mu \mathrm{M})$. Note the large increase in spontaneous bursting related to $I_{\mathrm{K}(\mathrm{ERG})}$ antagonism. D, D1, Rhythmic oscillations elicited on homosegmental (L5) lumbar roots by bath application of $5 \mu \mathrm{M}$ NMDA plus $10 \mu \mathrm{M} 5-\mathrm{HT}$. D2, Subsequent addition of E4031 increased burst period and duration. $\boldsymbol{E}$, The polar plot, constructed from the activity depicted in D1, shows that oscillations induced by NMDA and 5 -HT were not alternated between right and left L5 VRs and were poorly phase coupled. $\boldsymbol{F}$, When E4031 (1 $\mu \mathrm{M})$ was added, the polar plot (obtained from tracings in D2) confirms poor coupling between VR discharges, although synchronicity is improved. VRrL5, Right L5 VR; VRIL5, left L5 VR; VRrL2, right L2 VR; VRIL2, left L2 VR.

et al., 2005). Because coexpression of ERG and GABA has been detected in organotypic spinal slices (Furlan et al., 2005), we investigated whether a similar coexpression existed in transverse sections of E15 and E19.5 spinal cords in vivo. At E15, GABA immunoreactivity was localized mainly ventrally (Fig. 7A) (Avossa et al., 2003; Allain et al., 2004). Omission of the primary antibody produced only minimal fluorescence (Fig. $7 B$ ), indicating a lack of nonspecific staining. Note that the GABA antibody could not label motoneurons, suggesting an absence of nonselective immunoreactivity (supplemental Fig. 2, available at www. jneurosci.org as supplemental material). The expression of $m$-erg genes (investigated with NR-ISH) displayed a pattern similar to the one of GABA as exemplified with m-erg3 localization in the gray matter (Fig. 7E); NR-ISH performed with the sense probe gave no signal (Fig. $7 F$ ).

In colocalization studies, in which the same sections were processed for $m$-erg expression and GABA immunofluorescence, most of the stained cells, both ventrally (Fig. $7 C, G$ ) and dorsally (Fig. $7 D, H$ ), were double positive. Although only a few m-ergpositive cells were GABA negative (arrows), GABA-positive cells were always $m$-erg positive. Quantification of these observations (taken from E15 spinal cords) yielded an average of $15 \pm 2$ $m$-erg1a-, $7 \pm 1 \mathrm{~m}$-erg2-, and $13 \pm 3 \mathrm{~m}$-erg3-expressing cells per $2500 \mu \mathrm{m}^{2}$ (10 fields for each region in more than four sections) in the ventral region. Corresponding data for the dorsal region were $13 \pm 4,6 \pm 2$, and $11 \pm 3$. Most of the m-ergla-, m-erg2-, and $m$-erg-positive neurons were also GABA immunoreactive in the ventral or dorsal regions: 83 or $82 \%$ for $m$-ergla; 84 or $85 \%$ for $m$-erg 2 ; and 83 or $84 \%$ for $m$-erg3. In a similar set of experiments done on E19.5 spinal cords, the average number of $m$-erg-positive neurons was halved in the ventral region $(7 \pm 1$ cells per 2500 $\mu \mathrm{m}^{2}$ for $m$-erg1a, $3 \pm 1$ for $m$-erg2, and $6 \pm 2$ for $m$-erg 3$)$, whereas it remained unchanged in the dorsal region ( $12 \pm 2$ cells per $2500 \mu \mathrm{m}^{2}$ for $m$-erg1a, $6 \pm 1$ for $m$-erg2, and $10 \pm 1$ for $m$-erg3). Notwithstanding the local decrease in $m$-erg ventral neurons [in accordance with the report by Furlan et al. (2005)], residual $m$-erg-positive cells were usually immunoreactive for GABA (GABA colocalization: $80 \%$ for $m$-erg1a or $m$-erg2 and $81 \%$ for $m$-erg 3 ), like the neurons in the dorsal region (GABA colocalization: $81 \%$ for $m$-erg1a or $m$-erg 2 and $84 \%$ for $m$-erg 3 ).

\section{Discussion}

The main finding of the present study is the demonstration that, at an early stage of embryonic development of the spinal cord, the excitability of GABAergic ventral interneurons expressing $I_{\mathrm{K}-}$ (ERG), a recently discovered $\mathrm{K}^{+}$conductance, was largely raised by a selective $I_{\mathrm{K}(\mathrm{ERG})}$ blocker. Pharmacological inhibition of $I_{\mathrm{K}(\mathrm{ERG})}$ also led to stabilization and synchronization of VR patterns. All of these effects were lost shortly before birth. Thus, in analogy with brain networks (McBain and Fisahn, 2001), GABA-releasing interneurons appeared to control the output of large populations of spinal neurons via transiently expressed intrinsic $\mathrm{K}^{+}$currents. It seems likely that the developmental regulation of $I_{\mathrm{K}(\mathrm{ERG})}$ coincided with (and perhaps contributed to) the changing functional role of GABAergic interneurons from excitation to inhibition (Barbeau et al., 1999; Gao et al., 2001; Branchereau et al., 2002; Hanson and Landmesser, 2003).

\section{Organotypic spinal slices and spinal network development}

Organotypic spinal slices represent a biological model of segmental microcircuit development in which subsets of interneurons can be directly investigated at different growth times in vitro. Despite the absence of afferent and supraspinal inputs, which are important for the development of spinal circuits (Harris-Warrick and Marder, 1991; Nusbaum et al., 2001; Branchereau et al., 2002), this preparation represents a useful model for studying the dynamics of intrasegmental maturation processes that evidently rely on propriospinal neurons and circuits (Avossa et al., 2003; Rosato-Siri et al., 2004; Furlan et al., 2005; Fabbro et al., 2006). In particular, the ontogeny and functional development of GABAergic interneurons observed in vivo (Antal et al., 1994; Barbeau et al., 1999; Gao et al., 2001; Tran et al., 2003) is maintained in cultured spinal slices (Avossa et al., 2003; Furlan et al., 2005), validating the crucial importance of GABAergic connections for circuit assembly and activity (Barbeau et al., 1999). In the cultured spinal cord, ventral interneurons strongly, yet transiently, express a typical $I_{\mathrm{K}(\mathrm{ERG})}$ (Furlan et al., 2005) that controls membrane excitability (Chiesa et al., 1997; Sacco et al., 2003) in coincidence with the appearance of developmentally regulated GABAergic markers (Avossa et al., 2003; Furlan et al., 2005).

We show here for the first time that by blocking $I_{\mathrm{K}(\mathrm{ERG})}$, important physiological properties of certain GABAergic neurons in the ventral horn are changed only at a critical developmental 
time. Because of coexpression of GABA and $m$-erg genes, $I_{\mathrm{K} \text { (ERG) }}$ may be seen as a fingerprint of GABAergic neurons in spinal networks.

$I_{\mathrm{K}(\mathrm{ERG})}$ contributes to spike adaptation in GABAergic interneurons

Five different types of ventral interneurons in organotypic slices could be identified on the basis of their discharge patterns when synaptic transmission was blocked (Galante et al., 2000). In particular, the firing properties of the majority of ventral cells changed from adapting at early embryonic ages to tonic later in development. Such changes occurred in coincidence with the critical transformation of spontaneous activity from bursting to sporadic discharges (Whelan, 2003; Rosato-Siri et al., 2004). Previous studies reported similar neuronal firing classes in the ventral horn of the postnatal rodent spinal cord, with phasic versus tonic (or repetitive) cells distributed in a comparable proportion (Szucs et al., 2003; Theiss and Heckman, 2005).

Pharmacological block of $I_{\mathrm{K}(\mathrm{ERG})}$ by E4031 strongly suppressed frequency adaptation in spinal neurons at 7 DIV, indicating that this current was one mechanism to constrain firing patterns. Neurons with an electrophysiological phenotype other than adapting were insensitive to $I_{\mathrm{K}}$ (ERG) inhibition. Although it is possible that $I_{\mathrm{K}(\mathrm{ERG})}$ operated in synergy or competition with other membrane conductances, our functional results clearly indicate that the $I_{\mathrm{K}(\mathrm{ERG})}$-mediated modulation of discharge properties could be observed only during the time window (7 DIV) when this $\mathrm{K}^{+}$current is overwhelmingly expressed by ventral spinal interneurons (Avossa et al., 2003; Furlan et al., 2005).

Our results strongly suggest that the adapting cell type at 7 DIV could be mainly identified as a GABAergic phenotype. This notion is supported by several findings. Neurobiotin-filled adapting cells sensitive to the $I_{\mathrm{K}(\mathrm{ERG})}$ antagonist were also GABA positive, in keeping with our previous report in which all GABA immunopositive neurons were ERG positive as well (Furlan et al., 2005). The percentage of adapting cells in the present report is comparable with that reported for patched ventral interneurons at 7 DIV expressing the electrophysiological characteristics of $I_{\mathrm{K}(\mathrm{ERG})}$ (Furlan et al., 2005). Later (14 DIV), when GABA and ERG coexpression together with the presence of $m$-erg genes were found predominantly in the dorsal horn, the likelihood of detecting ventral adapting interneurons was largely reduced, and any cell in this category was E4031 insensitive. $m$-erg genes and GABA coexpression are similarly downregulated in the ventral spinal cord in vivo (Furlan et al., 2005).

During maturation in vitro, APs became sharper and more numerous, parameters that were never affected by the $I_{\mathrm{K}(\mathrm{ERG})}$ inhibitor. These data suggest that other voltage-gated conductances regulated the spike properties, including adaptation when $I_{\mathrm{K} \text { (ERG) }}$ itself was scantly expressed in the ventral horn (14 DIV).

Unlike the role of $I_{\mathrm{K}(\mathrm{ERG})}$ in controlling spike activity, the same current apparently did not contribute to resting membrane potential, which remained the same, despite the different $I_{\mathrm{K} \text { (ERG) }}$
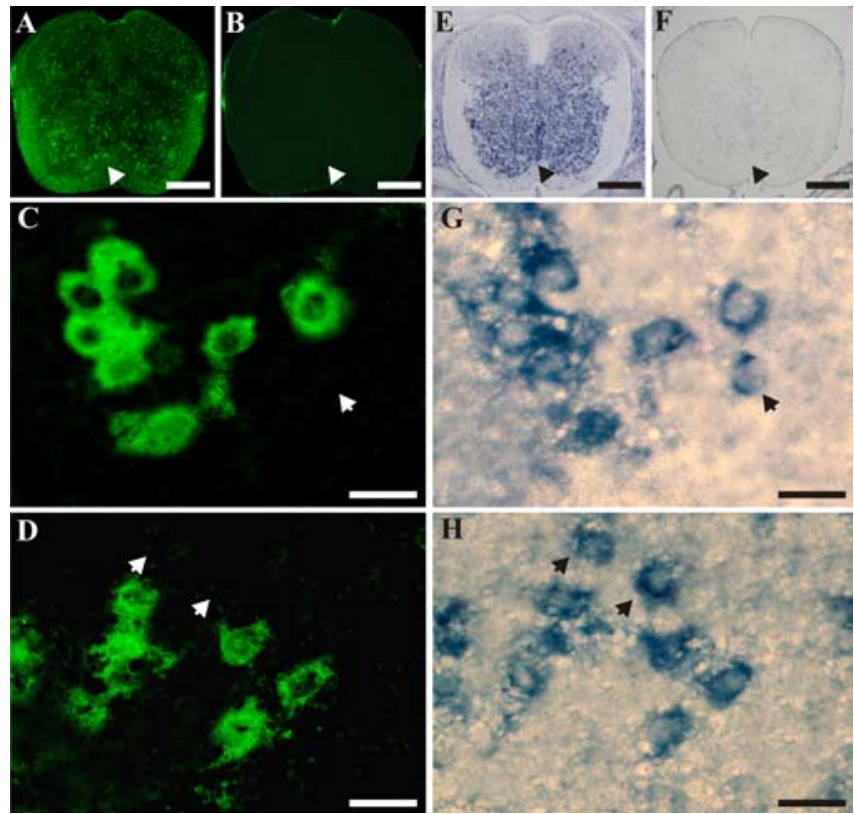

Figure 7. Coexpression of GABA and m-erg 3 in mouse embryonic spinal cord at E15. $A, B$, $G A B A$ immunoreactivity in sections incubated in the presence $(\boldsymbol{A})$ or absence $(\boldsymbol{B})$ of anti-GABA antibody (secondary antibody only). $\boldsymbol{E}, \boldsymbol{F}, m$-erg3 expression detected by NR-ISH with specific antisense $(\boldsymbol{E})$ and sense $(\boldsymbol{F})$ riboprobes. $\boldsymbol{C}, \boldsymbol{D}, \boldsymbol{G}, \boldsymbol{H}$, Detection in the same slice of $m$-erg3 $(\boldsymbol{C}, \boldsymbol{D})$ and $\operatorname{GABA}(\boldsymbol{G}, \boldsymbol{H})$ in the ventral $(\boldsymbol{C}, \boldsymbol{G})$ and dorsal $(\boldsymbol{D}, \boldsymbol{H})$ region of the E15 spinal cord. Note that most of $m$-erg3-expressing cells are GABA-positive cells ( $m$-erg3-positive, GABA-negative cells are indicated with arrows). Scale bars: $\boldsymbol{A}, \boldsymbol{B}, \boldsymbol{E}, \boldsymbol{F}, 200 \mu \mathrm{m} ; \boldsymbol{C}, \boldsymbol{D}, \mathbf{G}, \boldsymbol{H}, 18 \mu \mathrm{m}$. 
expression at 7 or $14 \mathrm{DIV}$. This observation is probably explained by the strong inactivation of $I_{\mathrm{K}(\mathrm{ERG})}$, which makes it unsuitable to contribute to the neuronal leak conductance.

\section{GABAergic neurons and the control of spinal network excitability}

In the immature spinal cord, as well as in other CNS areas, the ontogeny of inhibitory transmission is intriguing because of the temporal delay before the appearance of GABAergic and glycinergic inhibition (Antal et al., 1994; Barbeau et al., 1999; Gao et al., 2001; Tran et al., 2003; Nabekura et al., 2004; Allain et al., 2006). In the embryonic spinal cord, glycine progressively becomes the dominant force in driving more mature patterns of activity, such as coordinated locomotor-like bursts (Bonnot et al., 1998; Whelan et al., 2000). GABAergic interneurons appear to control the occurrence of spontaneous activity that, although not locomotor in nature, is seemingly necessary for normal motor network development (Barbeau et al., 1999; Gao et al., 2001; Moody and Bosma, 2005).

When synaptic transmission was left intact at 7 DIV, boosting GABAergic interneuron excitability by pharmacological removal of $I_{\mathrm{K}(\mathrm{ERG})}$ extended spontaneous bursts and increased overall firing. This observation accords with the demonstrated loss of adaptation in the presence of the $I_{\mathrm{K}(\mathrm{ERG})}$ antagonist. It is feasible to propose that, at this early stage, sustained firing of GABAergic neurons prolonged bursting and interburst firing. The slower period of bursting may be accounted for by the stronger coactivation of mechanisms like the $\mathrm{Na}^{+}-\mathrm{K}^{+}$pump (Ballerini et al. 1997; Darbon et al., 2003), which usually restrains bursting frequency.

\section{Blocking $I_{K(E R G)}$ in the isolated spinal cord reveals a role for this conductance in rhythmic pattern generation}

In E15 isolated spinal cord preparations, E4031 triggered repeated asynchronous discharges that we may interpret, on the basis of current data from organotypic cultures, as a consequence of depressing firing adaptation in certain GABAergic interneurons. When network excitability was largely raised by coapplication of NMDA and 5-HT (Vinay et al., 2002), irregular patterns emerged from VRs, confirming that, at this stage, there was no rhythm coordination at the segmental (or intersegmental) level (Whelan, 2003; Kiehn, 2006). E4031 converted this activity into more regular, longer bursts appearing synchronously between segments. The simplest interpretation is that E4031 facilitated the firing of certain GABAergic interneurons to recruit network neurons in a more coherent pattern. Lack of discharge alternation, however, indicated that there was no electrophysiological evidence for synaptic inhibition mediated by GABA (Whelan, 2003; Kiehn, 2006) either before or after applying E4031. Just before birth, when alternated locomotor patterns appear and rely on GABA- and glycine-mediated inhibition within the ventral horn area (Bonnot et al., 1998; Whelan et al., 2000), E4031 did not affect either pattern generation or rhythmicity, in accordance with the predominantly dorsal localization of the $m$-erg gene and GABA-positive cells. The number of ventral interneurons expressing $m$-erg was reduced, although they usually retained GABA immunoreactivity. Future studies will be necessary to ascertain the properties of $I_{\mathrm{K}(\mathrm{ERG})}$ in ventral interneurons of the isolated spinal cord and to compare them with those observed in organotypic slices.

\section{Functional significance}

The E4031 efficacy to modulate spinal network excitability restricted to an early stage of development, plus m-erg gene and GABA coexpression, suggested that the full operation of $I_{\mathrm{K}(\mathrm{ERG})}$ constrained the excitatory action of ventral horn GABAergic interneurons. In analogy with other cell models such as neuroblastoma cells (Arcangeli et al., 1995, 1998) or quail neural crest cells (Arcangeli et al., 1997; Crociani et al., 2000), it appears that ERG conductances consistently, yet only transiently, modulate neuronal excitability. Later in development, this braking action on excitability is replaced by other $\mathrm{K}^{+}$currents (McBain and Fisahn, 2001). In addition, membrane ERG channels may play a role in axon guidance and network formation by GABAergic interneurons because ERG can interact with signaling molecules like the adhesive receptors of the integrin family (Arcangeli et al., 2004; Cherubini et al., 2005). According to this hypothesis, the timedependent expression of ERG channels would be downregulated once GABA becomes inhibitory.

\section{References}

Allain AE, Bairi A, Meyrand P, Branchereau P (2004) Ontogenic changes of the GABAergic system in the embryonic mouse spinal cord. Brain Res 1000:134-147.

Allain AE, Bairi A, Meyrand P, Branchereau P (2006) Expression of the glycinergic system during the course of embryonic development in the mouse spinal cord and its colocalization with GABA immunoreactivity. J Comp Neurol 496:832-846.

Alvarez FJ, Jonas PC, Sapir T, Hartley R, Berrocal MC, Geiman EJ, Todd AJ, Goulding M (2005) Postnatal phenotype and localization of spinal cord V1 derived interneurons. J Comp Neurol 493:177-192.

Antal M, Berki AC, Horvath L, O’Donovan MJ (1994) Developmental changes in the distribution of gamma-aminobutyric acid-immunoreactive neurons in the embryonic chick lumbosacral spinal cord. J Comp Neurol 343:228-236.

Arcangeli A, Bianchi L, Becchetti A, Faravelli L, Coronnello M, Mini E, Olivotto M, Wanke E (1995) A novel inward-rectifing $\mathrm{K}^{+}$current with a cell cycle dependence governs the resting potential of mammalian neuroblastoma cells. J Physiol (Lond) 489:455-471.

Arcangeli A, Rosati B, Cherubini A, Crociani O, Fontana L, Ziller C, Wanke E, Olivotto M (1997) HERG- and IRK-like inward currents are sequentially expressed during neuronal development of neural crest cells and their derivates. Eur J Neurosci 9:2596-2604.

Arcangeli A, Rosati B, Cherubini A, Crociani O, Fontana L, Passani B, Wanke E, Olivotto M (1998) Long-term exposure to retinoic acid induces the expression of IRK1 channels in HERG channel-endowed neuroblastoma cells. Biochem Biophys Res Commun 244:706-711.

Arcangeli A, Becchetti A, Cherubini A, Crociani O, Defilippi P, Guasti L, Hofmann G, Pillozzi S, Olivotto M, Wanke E (2004) Physical and functional interaction between integrins and hERG potassium channels. Biochem Soc Trans 32:826-827.

Avossa D, Rosato-Siri MD, Mazzarol F, Ballerini L (2003) Spinal circuits formation: a study of developmentally regulated markers in organotypic cultures of embryonic mouse spinal cord. Neuroscience 122:391-405.

Avossa D, Grandolfo M, Mazzarol F, Zatta M, Ballerini L (2006) Early signs of motoneuron vulnerability in a disease model system: characterization of transverse slice cultures of spinal cord isolated from embryonic ALS mice. Neuroscience 138:1179-1194.

Ballerini L, Galante M (1998) Network bursting by organotypic spinal slice cultures in the presence of bicuculline and/or strychnine is developmentally regulated. Eur J Neurosci 10:2871-2879.

Ballerini L, Bracci E, Nistri A (1997) Pharmacological block of the electrogenic pump disrupts rhythmic bursting induced by strychnine and bicuculline in the neonatal rat spinal cord. J Neurophysiol 77:17-23.

Ballerini L, Galante M, Grandolfo M, Nistri A (1999) Generation of rhythmic patterns of activity by ventral interneurones in rat organotypic spinal slice culture. J Physiol (Lond) 517:459-475.

Barbeau H, McCrea DA, O’Donovan MJ, Rossignol S, Grill WM, Lemay MA (1999) Tapping into spinal circuits to restore motor function. Brain Res Brain Res Rev 30:27-51.

Bauer CK, Schwarz JR (2001) Physiology of EAG K ${ }^{+}$channels. J Membr Biol 182:1-15.

Ben-Ari Y (2002) Excitatory actions of GABA during development: the nature of the nurture. Nat Rev Neurosci 3:728-739.

Bonnot A, Morin D, Viala D (1998) Genesis of spontaneous rhythmic mo- 
tor patterns in the lumbosacral spinal cord of neonate mouse. Brain Res Dev Brain Res 108:89-99.

Branchereau P, Chapron J, Meyrand P (2002) Descending 5-hydroxytryptamine raphe inputs repress the expression of serotonergic neurons and slow the maturation of inhibitory systems in mouse embryonic spinal cord. J Neurosci 22:2598-2606.

Butt SJ, Lebret JM, Kiehn O (2002) Organization of left-right coordination in the mammalian locomotor network. Brain Res Rev 40:107-117.

Caserta MT, Barker JL (1994) Development of the GABAergic phenotype in murine spinal cord-dorsal root ganglion cultures. Int J Dev Neurosci 12:753-765.

Cherubini A, Hofmann G, Pillozzi S, Guasti L, Crociani O, Cilia E, Di Stefano P, Degani S, Balzi M, Olivotto M, Wanke E, Becchetti A, Defilippi P, Wymore R, Arcangeli A (2005) Human ether-a-go-go-related gene 1 channels are physically linked to beta 1 integrins and modulate adhesiondependent signaling. Mol Biol Cell 16:2972-2983.

Chiesa N, Rosati B, Arcangeli A, Olivotto M, Wanke E (1997) A novel role for HERG $\mathrm{K}^{+}$channels: spike-frequency adaptation. J Physiol (Lond) 501:313-318.

Crociani O, Cherubini A, Piccini E, Polvani S, Costa L, Fontana L, Hofmann G, Rosati B, Wanke E, Olivotto M, Arcangeli A (2000) erg gene(s) expression during development of the nervous and muscular system of quail embryos. Mech Dev 95:239-243.

Darbon P, Tscherter A, Yvon C, Streit J (2003) Role of the electrogenic Na/K pump in disinhibition-induced bursting in cultured spinal networks. J Neurophysiol 90:3119-3129.

Drew T, Doucet S (1991) Application of circular statistics to the study of neuronal discharge during locomotion. J Neurosci Methods 38:171-181.

Fabbro A, Pastore B, Nistri A, Ballerini L (2006) Activity-independent intracellular $\mathrm{Ca}(2+)$ oscillations are spontaneously generated by ventral spinal neurons during development in vitro. Cell Calcium, in press.

Feldblum S, Anoal M, Lapsher S, Dumoulin A, Privat A (1998) Partial deafferentation of the developing rat spinal cord delays the spontaneous repression of GAD67 mRNAs in spinal cells. Perspect Dev Neurobiol 5:131-143.

Furlan F, Guasti L, Avossa D, Becchetti A, Cilia E, Ballerini L, Arcangeli A (2005) Interneurons transiently express the $\mathrm{ERG} \mathrm{K}^{+}$channels during development of mouse spinal networks in vitro. Neuroscience 135:1179-1192.

Galante M, Nistri A, Ballerini L (2000) Opposite changes in synaptic activity of organotypic rat spinal cord cultures after chronic block of AMPA/ kainate or glycine and GABAA receptors. J Physiol (Lond) 523:639-651.

Gao BX, Stricker C, Ziskind-Conhaim L (2001) Transition from GABAergic to glycinergic synaptic transmission in newly formed spinal networks. J Neurophysiol 86:492-502.

Hanson MG, Landmesser LT (2003) Characterization of the circuits that generate spontaneous episodes of activity in early embryonic mouse spinal cord. J Neurosci 15:587-600.

Harris-Warrick RM, Marder E (1991) Modulation of neural networks for behaviour. Annu Rev Neurosci 14:39-57.

Hirdes W, Schweizer M, Schuricht KS, Guddat SS, Wulfsen I, Bauer CK, Schwarz JR (2005) Fast erg $\mathrm{K}^{+}$currents in rat embryonic serotonergic neurones. J Physiol (Lond) 564:33-49.

Jessel TM (2000) Neuronal specification in the spinal cord: inductive signals and transcriptional codes. Nat Rev Genet 1:20-29.

Kiehn O (2006) Locomotor circuits in the mammalian spinal cord. Annu Rev Neurosci 29:279-306.

Kiehn O, Kjaerulff O (1998) Distribution of central pattern generators for rhythmic motor outputs in the spinal cord of limbed vertebrates. Ann NY Acad Sci 860:110-129.

Kjaerulff O, Kiehn O (1996) Distribution of networks generating and coordinating locomotor activity in the neonatal rat spinal cord in vitro: a lesion study. J Neurosci 16:5777-5794.

Lu VB, Moran TD, Balasubramanyan S, Alier KA, Dryden WF, Colmers WF, Smith PA (2006) Substantia gelatinosa neurons in defined-medium organotypic slice culture are similar to those in acute slices from young adult rats. Pain 121:261-275.

McBain CJ, Fisahn A (2001) Interneurons unbound. Nat Rev Neurosci 2:11-23.

Moody WJ, Bosma M (2005) Ion channel development, spontaneous activity, and activity-dependent development in nerve and muscle cells. Physiol Rev 85:883-941.

Nabekura J, Katsurabayashi S, Kakazu Y, Shibata S, Matsubara A, Jinno S,
Mizoguchi Y, Sasaki A, Ishibashi H (2004) Developmental switch from GABA to glycine release in single central synaptic terminals. Nat Neurosci 7:17-23.

Nakayama K, Nishimaru H, Kudo N (2002) Basis of changes in left-right coordination of rhythmic motor activity during development in the rat spinal cord. J Neurosci 22:10388-10398.

Nishimaru H, Iizuka M, Ozaki S, Kudo N (1996) Spontaneous motoneuronal activity mediated by glycine and GABA in the spinal cord of rat fetuses in vitro. J Physiol (Lond) 497:131-143.

Nistri A, Ostroumov K, Sharifullina E, Taccola G (2006) Tuning and playing a motor rhythm: how metabotropic glutamate receptors orchestrate generation of motor patterns in the mammalian central nervous system. J Physiol (Lond) 572:323-334.

Nusbaum MP, Blitz DM, Swensen AM, Wood D, Marder E (2001) The roles of co-transmission in neural network modulation. Trends Neurosci 24:146-154.

Owens DF, Kriegstein AR (2002) Is there more to GABA than synaptic inhibition? Nat Rev Neurosci 3:715-727.

Papa M, Boscia F, Canitano A, Castaldo P, Sellitti S, Annunziato L, Taglialatela M (2003) Expression pattern of the ether-a-go-go-related (ERG) $\mathrm{K}^{+}$channel-encoding genes ERG1, ERG2, and ERG3 in the adult rat central nervous system. J Comp Neurol 466:119-135.

Phelps PE, Alijani A, Tran TS (1999) Ventrally located commissural neurons express the GABAergic phenotype in developing rat spinal cord. J Comp Neurol 409:285-298.

Prescott SA, de Koninck Y (2002) Four cell types with distinctive membrane properties and morphologies in lamina I of the spinal dorsal horn of the adult rat. J Physiol (Lond) 539:817-836.

Rosato-Siri MD, Grandolfo M, Ballerini L (2002) Activity-dependent modulation of GABAergic synapses in developing rat spinal networks in vitro. Eur J Neurosci 16:2123-2135.

Rosato-Siri MD, Zoccolan D, Furlan F, Ballerini L (2004) Interneurone bursts are spontaneously associated with muscle contractions only during early phases of mouse spinal network development: a study in organotypic cultures. Eur J Neurosci 20:2697-2710.

Sacco T, Bruno A, Wanke E, Tempia F (2003) Functional roles of an ERG current isolated in cerebellar Purkinje neurons. J Neurophysiol 90:1817-1828.

Schwarz JR, Bauer CK (1999) The ether-a-go-go-related gene K(+) current: functions of a strange inward rectifier. News Physiol Sci 14:135-142.

Schwarz JR, Bauer CK (2004) Functions of erg K channels in excitable cells. J Cell Mol Med 8:22-30.

Somogyi R, Wen X, Ma W, Barker JL (1995) Developmental kinetics of GAD family mRNAs parallel neurogenesis in the rat spinal cord. J Neurosci 15:2575-2591.

Streit J, Spenger C, Lucher HR (1991) An organotypic spinal cord dorsal root ganglion skeletal muscle coculture of embryonic rat. II. Functional evidence for the information of spinal reflex arcs in vitro. Eur J Neurosci 3:1054-1068.

Szucs P, Odeh F, Szokol K, Antal M (2003) Neurons with distinctive firing patterns, morphology and distribution in laminae V-VII of the neonatal rat lumbar spinal cord. Eur J Neurosci 17:537-544.

Taccola G, Nistri A (2004) Low micromolar concentrations of 4 -aminopyridine facilitate fictive locomotion expressed by the rat spinal cord in vitro. Neuroscience 126:511-520.

Theiss RD, Heckman CJ (2005) Systematic variation in effects of serotonin and norepinephrine on repetitive firing properties of ventral horn neurons. Neuroscience 134:803-815.

Tran TS, Alijani A, Phelps PE (2003) Unique developmental patterns of GABAergic neurons in rat spinal cord. J Comp Neurol 456:112-126.

Vinay L, Brocard F, Clarac F, Norreel JC, Pearlstein E, Pflieger JF (2002) Development of posture and locomotion: an interplay of endogenously generated activities and neurotrophic actions by descending pathways. Brain Res Rev 40:118-129.

Whelan P, Bonnot A, O'Donovan MJ (2000) Properties of rhythmic activity generated by the isolated spinal cord of the neonatal mouse. J Neurophysiol 84:2821-2833.

Whelan PJ (2003) Developmental aspects pf spinal locomotor function: insight from using the in vitro mouse spinal cord preparation. J Physiol (Lond) 553:695-706.

Wimmers S, Wulfsen I, Bauer CK, Schwarz JR (2001) erg1, erg2 and erg3 K channel subunits are able to form heteromultimers. Pflügers Arch 441: 450-455. 